\title{
PRODUCTION OF ALL THE $r$-PROCESS NUCLIDES IN THE DYNAMICAL EJECTA OF NEUTRON STAR MERGERS
}

\author{
Shinya Wanajo ${ }^{1}$, Yuichiro Sekiguchi ${ }^{2}$, Nobuya Nishimura ${ }^{3}$, Kenta KiUchi $^{2}$, \\ Koutarou Kyutoku ${ }^{4}$, and Masaru Shibata ${ }^{2}$ \\ 1 iTHES Research Group, RIKEN, Wako, Saitama 351-0198, Japan; shinya.wanajo@ riken.jp \\ ${ }^{2}$ Yukawa Institute for Theoretical Physics, Kyoto University, Kyoto 606-8502, Japan \\ 3 Astrophysics, EPSAM, Keele University, Keele ST5 5BG, UK \\ ${ }^{4}$ Department of Physics, University of Wisconsin-Milwaukee, P.O. Box 413, Milwaukee, WI 53201, USA \\ Received 2014 February 28; accepted 2014 June 9; published 2014 June 26
}

\begin{abstract}
Recent studies suggest that binary neutron star (NS-NS) mergers robustly produce heavy $r$-process nuclei above the atomic mass number $A \sim 130$ because their ejecta consist of almost pure neutrons (electron fraction of $\left.Y_{\mathrm{e}}<0.1\right)$. However, the production of a small amount of the lighter $r$-process nuclei $(A \approx 90-120)$ conflicts with the spectroscopic results of $r$-process-enhanced Galactic halo stars. We present, for the first time, the result of nucleosynthesis calculations based on the fully general relativistic simulation of a NS-NS merger with approximate neutrino transport. It is found that the bulk of the dynamical ejecta are appreciably shock-heated and neutrino processed, resulting in a wide range of $Y_{\mathrm{e}}(\approx 0.09-0.45)$. The mass-averaged abundance distribution of calculated nucleosynthesis yields is in reasonable agreement with the full-mass range $(A \approx 90-240)$ of the solar $r$-process curve. This implies, if our model is representative of such events, that the dynamical ejecta of NS-NS mergers could be the origin of the Galactic $r$-process nuclei. Our result also shows that radioactive heating after $\sim 1$ day from the merging, which gives rise to $r$-process-powered transient emission, is dominated by the $\beta$-decays of several species close to stability with precisely measured half-lives. This implies that the total radioactive heating rate for such an event can be well constrained within about a factor of two if the ejected material has a solar-like $r$-process pattern.
\end{abstract}

Key words: nuclear reactions, nucleosynthesis, abundances - stars: abundances - stars: neutron

Online-only material: color figures

\section{INTRODUCTION}

The astrophysical site of the $r$-process, the rapid neutroncapture process that makes half of all elements heavier than iron, remains a long-standing mystery of nucleosynthesis. Recently, compact binary mergers (CBMs) of double neutron star (NS-NS) and black hole-neutron star (BH-NS) systems have received considerable attention as possible sources of $r$-process nuclei (Lattimer \& Schramm 1974; Symbalisty \& Schramm 1982; Eichler et al. 1989; Meyer 1989; Freiburghaus et al. 1999) for the following reasons.

First, radioactively powered "kilonova" emission from the $r$-processed ejecta can be a promising electromagnetic counterpart to the gravitational-wave signal from a CBM event (Li \& Paczyński 1998; Metzger et al. 2010; Goriely et al. 2011; Kasen et al. 2013; Barnes \& Kasen 2013; Tanaka \& Hotokezaka 2013; Grossman et al. 2014). The possible identification of a kilonova associated with the Swift GRB 130603B (Berger et al. 2013; Tanvir et al. 2013) also indicates that CBMs are the progenitors of short-duration gamma-ray bursts and the sources of $r$-process elements (Hotokezaka et al. 2013b; Tanaka et al. 2014).

Another reason is that core-collapse supernovae (CCSNe; in particular proto-NS wind), which have been believed to be a promising source of the $r$-process nuclei, are found to provide only marginal conditions for creating elements beyond iron (Martínez-Pinedo et al. 2012; Roberts et al. 2012; Fischer et al. 2012). Nucleosynthesis studies with such physical environments confirm that CCSNe produce elements only up to the atomic mass number $A \sim 110$ (Wanajo et al. 2011; Wanajo 2013). One possible exception could be the scenario of (still hypothetical) rapidly rotating, strongly magnetized CCSN cores (Winteler et al. 2012).
Recently, Goriely et al. (2011) and Bauswein et al. (2013) have explored nucleosynthesis based on the approximate (conformally flat spatial metric) general-relativistic (GR) simulations of NS-NS mergers. They found that the ejecta had extremely low electron fractions $\left(Y_{\mathrm{e}}<0.1\right)$, which led to fission recycling and thus robust production of only heavy $r$-process nuclei with $A \gtrsim 130$. Similar results were obtained from the Newtonian simulations of NS-NS and BH-NS mergers by Roberts et al. (2011), Korobkin et al. (2012), Rosswog et al. (2014).

The production of a smaller amount of the lighter $r$-process nuclei $(A \approx 90-120)$ conflicts, however, with the recent spectroscopic results of Galactic halo stars (Sneden et al. 2008; Siqueira Mello et al. 2014). That is, the so-called "universality" of the (solar-like) $r$-process pattern, first identified for $Z \gtrsim 56$ $(A \gtrsim 140)$, persists down to $Z \sim 38(A \sim 90)$ within about a factor of two. There has been no sign of nucleosynthetic events creating the nuclei exclusively with $A \gtrsim 130$. Contribution from, e.g., the subsequent BH accretion-torus wind (Surman et al. 2008; Wanajo \& Janka 2012; Fernández \& Metzger 2013) might solve this problem.

In this Letter, we report our first result of nucleosynthesis study based on the full GR, approximate neutrino transport simulation of a NS-NS merger. The GR effects, which are crucial for the dynamical evolutions of merger ejecta as pointed out by Hotokezaka et al. (2013a), were not fully taken into account in previous studies. Moreover, neutrino transport that can affect the ejecta $Y_{\mathrm{e}}$ is neglected in all previous studies (except for the twodimensional Newtonian simulation by Dessart et al. 2009, without nucleosynthesis calculations). Our NS-NS merger model is described in Section 2. The subsequent nucleosynthesis result is presented in Section 3. The radioactive heating rates 
(relevant for kilonova emission) are also obtained from the nucleosynthesis calculations (Section 4).

\section{MERGER MODEL}

The hydrodynamical evolution of a NS-NS merger is followed with a recently developed three-dimensional (3D) fullGR code (Y. Sekiguchi et al. 2014, in preparation), which is updated from the previous version (Sekiguchi 2010; Sekiguchi et al. 2011a, 2011b). Neutrino transport is taken into account based on the Thorne's moment scheme (Thorne 1981; Shibata et al. 2011) with a closure relation. For neutrino heating, absorption on free nucleons is considered. The gravitational masses (in isolation) are taken to be $1.3 M_{\odot}$ for both NSs.

We adopt an equation of state (EOS) of dense matter developed in Steiner et al. (2013, SFHo), which has a maximum NS mass sufficiently greater than the largest well-measured mass $\left(\approx 2 M_{\odot}\right.$, Demorest et al. 2010; Antoniadis et al. 2013). This EOS gives the radius $\approx 12 \mathrm{~km}$ for a cold NS, which is in the range constrained from nuclear experiments, nuclear theory, and astrophysical observations, $10.7-13.1 \mathrm{~km}$ for a $1.4 M_{\odot} \mathrm{NS}$ (Lattimer \& Lim 2013). Note that the EOS of Shen et al. (1998) adopted in many previous simulations gives $\approx 14.5 \mathrm{~km}$ for a $1.4 M_{\odot} \mathrm{NS}$, which is substantially greater than the upper bound of this constraint.

At the beginning of simulation $(t=0)$, each NS consists of matter with $Y_{\mathrm{e}} \approx 0.06$ in the (neutrino-less) $\beta$-equilibrium with a constant temperature of $0.1 \mathrm{MeV}$. The background medium is placed with the same temperature, density decreasing from $10^{5} \mathrm{~g} \mathrm{~cm}^{-3}$ (in the central region) to $10^{3} \mathrm{~g} \mathrm{~cm}^{-3}$, and $Y_{\mathrm{e}}=0.46$. The merging of NSs starts at $t \sim 3 \mathrm{~ms}$ with increasing density at the origin of the coordinate axis, $\rho_{0}$ (Figure 1). This leads to the steep rises of masses $(t \sim 5.5 \mathrm{~ms}$ ) outside $150 \mathrm{~km}$ (from the center) coming from the contact interface region.

A hypermassive NS (HMNS) forms at $t \sim 4.5 \mathrm{~ms}$. The second phase of mass ejection follows in response to the interaction between the inner atmospheric material (originating from the shear interface) and the rapidly rotating, quasi-radially oscillating HMNS (from $t \sim 7.5 \mathrm{~ms}$ ). We find that the total ejecta mass is dominated $(\sim 60 \%)$ by this second phase. The simulation ends at $t=13.7 \mathrm{~ms}$ with the distributions of density, temperature, $Y_{\mathrm{e}}$, and entropy (per nucleon; $S / k_{\mathrm{B}}, k_{\mathrm{B}}$ is Boltzmann's constant) shown in Figure $2 .{ }^{5}$ At this time, the bulk of ejecta (total mass of $M_{\mathrm{ej}} \approx 0.01 M_{\odot}$ ) are freely expanding with the velocities $\sim(0.1-0.3) c$ ( $c$ is the speed of light).

The behavior of mass ejection described here is in qualitative agreement with the previous full GR (Hotokezaka et al. 2013a, for soft EOSs) and approximate GR (Bauswein et al. 2013, for the same SFHo EOS) works. As pointed out in these studies, the mass ejection is due to shock-heating and tidal torque; neutrinoheating plays a subdominant role.

As the HMNS forms, temperature near its surface gets as high as $\sim 10 \mathrm{MeV}(\sim 100 \mathrm{GK})$, giving rise to copious $e^{-} e^{+}$pairs that activate the weak interactions $n+e^{+} \rightarrow \bar{v}_{e}+p, p+e^{-} \rightarrow v_{e}+n$, and their inverses. The $e^{+}$and $v_{e}$ captures convert some part of neutrons to protons; the ejecta $Y_{\mathrm{e}}$ values increase from the initial low values. ${ }^{6}$ The first outgoing ejecta from the contact interface region are away from the HMNS when it forms and

\footnotetext{
5 Animations of the simulation are available from http://cosnucs.riken.jp/movie.html.

6 The fast moving NSs and subsequent merger ejecta in the background medium make shocks that can increase temperature and thus $Y_{\mathrm{e}}$. However, the mass suffering from these artifacts is negligibly small compared to the total ejecta mass (Figure 1).
}
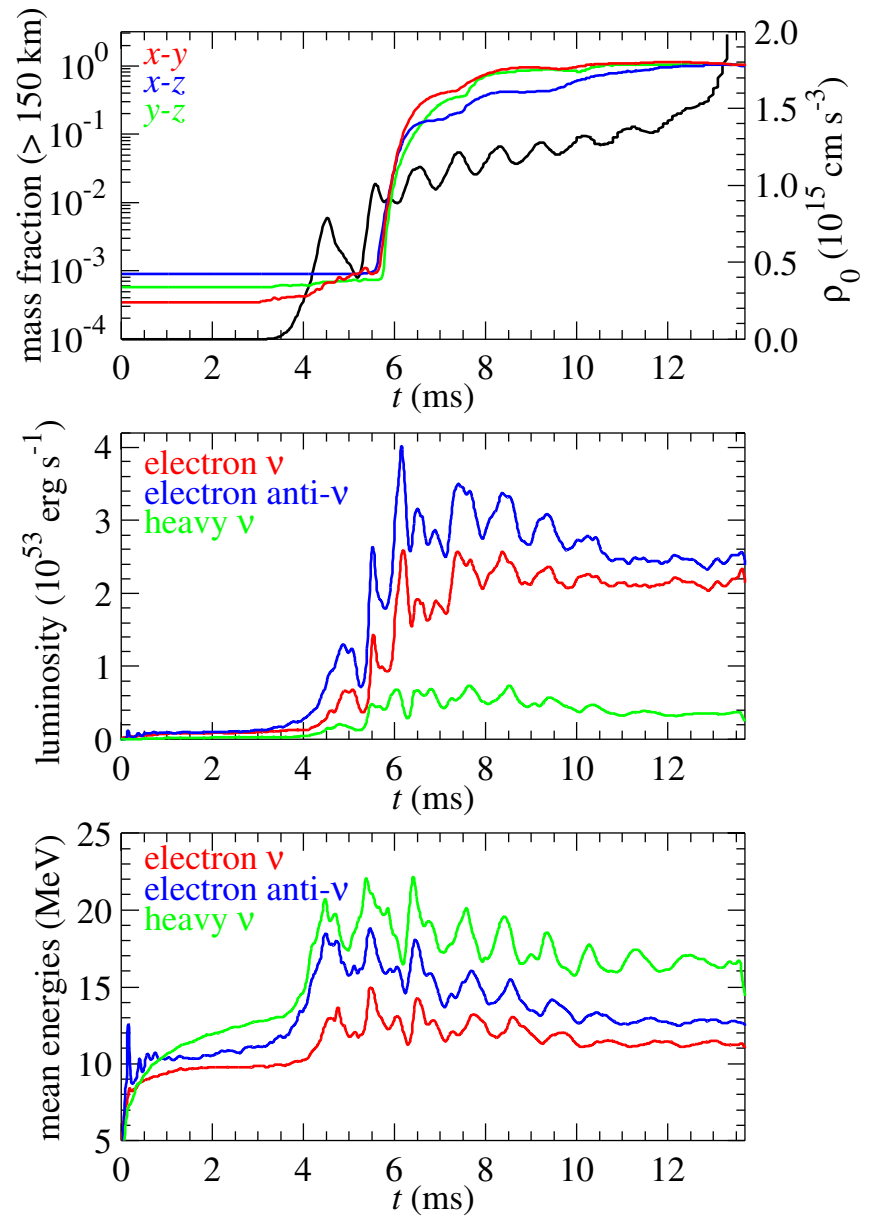

Figure 1. Temporal evolutions of ejecta mass fractions outside $150 \mathrm{~km}$ from the origin of the coordinate axis for the $x-y, x-z$, and $y-z$ planes in the $(2000 \mathrm{~km})^{3}$ cube (see Figure 2 ; with the width $\approx 13 \mathrm{~km}$ for each plane). The ejecta mass ratio at the end of simulation is $\sim 5: 2: 3$ for these planes. The masses at $t=0$ are due to the background medium, the fractions of which are sufficiently small compared to the total masses. Also shown is the temporal evolution of density at the origin. The middle and bottom panels display, respectively, the luminosities and angle-averaged mean energies for $v_{e}, \bar{v}_{e}$, and heavy-lepton neutrinos. Note that the neutrinos of $\sim 10 \mathrm{MeV}$ at $t \lesssim 4 \mathrm{~ms}$ are unimportant because of the low luminosities.

(A color version of this figure is available in the online journal.)

thus neutrino capture is subdominant. As a result, the $Y_{\mathrm{e}}$ values are relatively low $(\sim 0.1-0.2$; blue-cyan spiral arms in Figure 2). The outer ejecta with higher $Y_{\mathrm{e}}(\sim 0.2-0.3)$ are unimportant in the total ejecta mass because of their low densities.

In the second phase of mass ejection, neutrinos coming from the HMNS surface play a crucial role. The luminosities and mean energies are only slightly greater for $\bar{v}_{e}$ than those for $v_{e}$ (Figure 1). The asymptotic $Y_{\mathrm{e}}$ (after sufficient time) with these values is expected to be $Y_{\mathrm{e}, \mathrm{a}} \sim 0.5$ (e.g., Equation (77) in Qian \& Woosley 1996). However, neutrino absorption in the fast outgoing ejecta freezes before $Y_{\mathrm{e}}$ reaches $Y_{\mathrm{e}, \mathrm{a}}$, resulting in $Y_{\mathrm{e}} \sim 0.3-0.4$ (yellow-orange spiral arms in Figure 2).

The ejecta mass distributions in $Y_{\mathrm{e}}$ and $S / k_{\mathrm{B}}$ at the end of the simulation are displayed in Figure 3 for the $x-y, x-z$, and $y-z$ planes. We find that the $Y_{\mathrm{e}}$ values widely vary between 0.09 and 0.45 with greater amounts for higher $Y_{\mathrm{e}}$, in which the initial $\beta$-equilibrium values $(\approx 0.06)$ have gone. Non-orbital ejecta have higher $Y_{\mathrm{e}}$ values because of the shock-heated matter escaping to the low-density polar regions (Hotokezaka et al. 2013a). The shock heating results in $S / k_{\mathrm{B}}$ up to $\approx 26$ and 50 

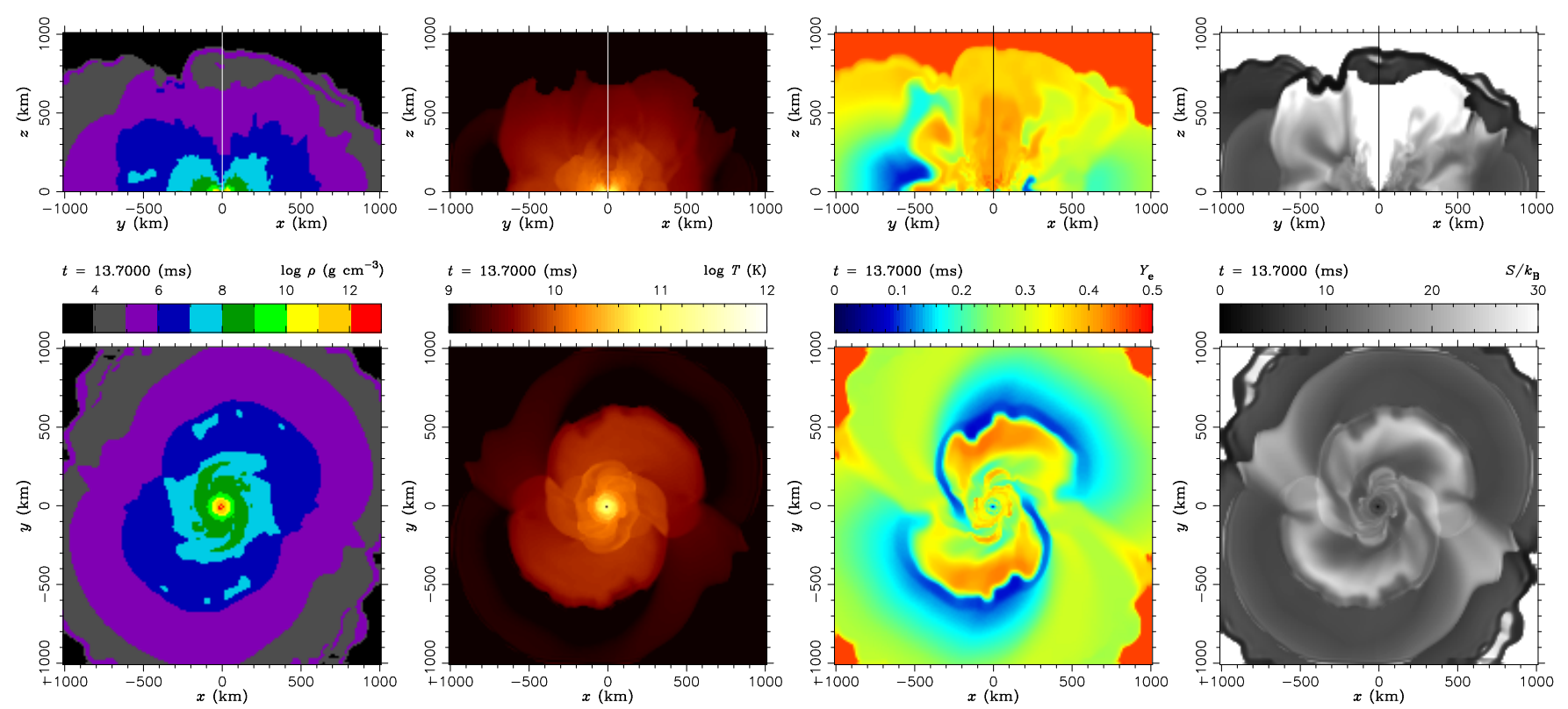

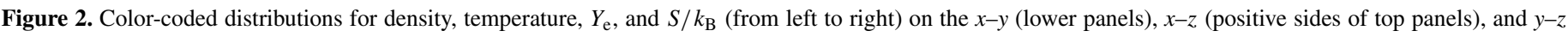
(negative sides of top panels) planes at the end of simulation.

(A color version of this figure is available in the online journal.)
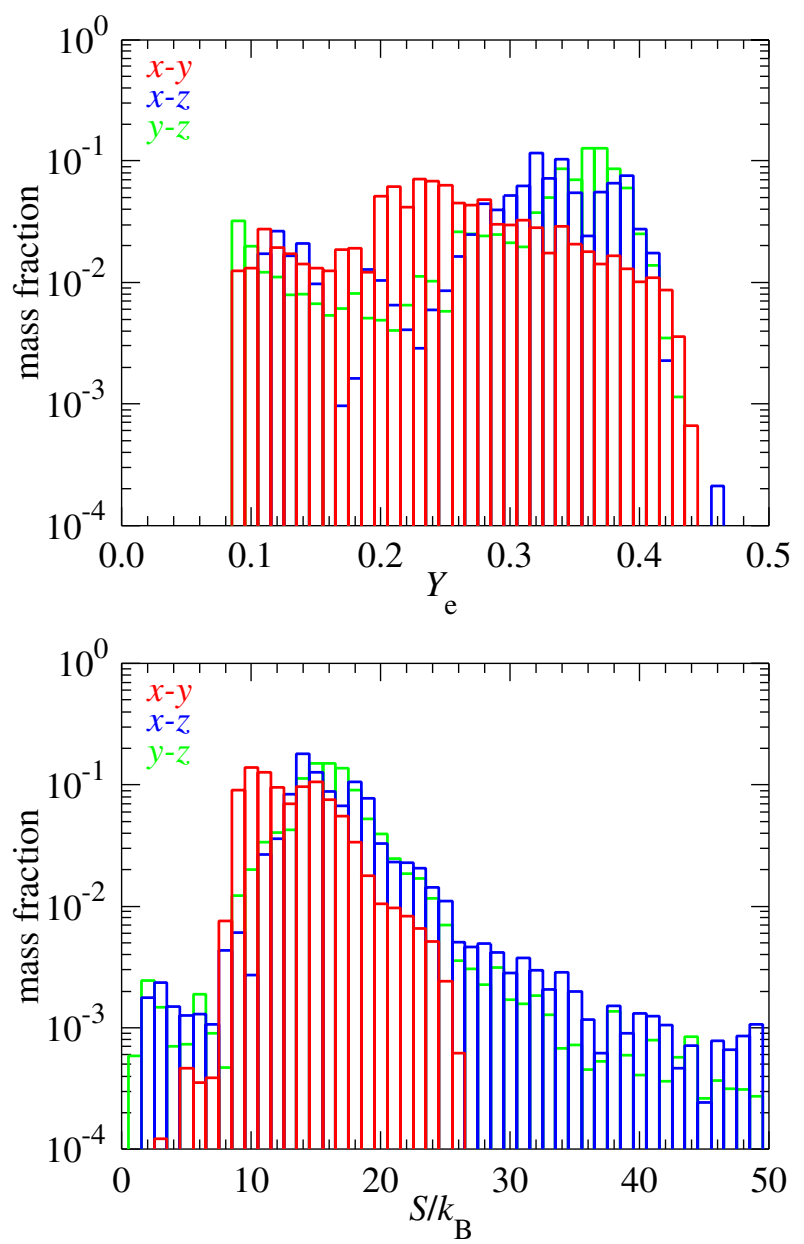

Figure 3. Mass fractions outside $150 \mathrm{~km}$ from the center vs. $Y_{\mathrm{e}}$ (top) and $S / k_{\mathrm{B}}$ (bottom) at the end of simulation for the $x-y, x-z$, and $y-z$ planes. The widths of $Y_{\mathrm{e}}$ and $S / k_{\mathrm{B}}$ are chosen to be $\Delta Y_{\mathrm{e}}=0.01$ and $\Delta S / k_{\mathrm{B}}=1$, respectively. (A color version of this figure is available in the online journal.) for the orbital and non-orbital planes, respectively (with higher values for higher $Y_{\mathrm{e}}$ ), which are sizably greater than those in Goriely et al. (2011, $\left.S / k_{\mathrm{B}} \sim 1-3\right)$ with the Shen's EOS.

\section{THE $r$-PROCESS}

The nucleosynthesis analysis makes use of the thermodynamic trajectories of the ejecta particles traced on the orbital plane. A representative particle is chosen from each $Y_{\mathrm{e}}$-bin (from $Y_{\mathrm{e}}=0.09$ to 0.44 with the interval of $\Delta Y_{\mathrm{e}}=0.01$ (Figure 3). For simplicity, we analyze only the $x-y$ components because of the dominance of the ejecta masses close to the orbital plane. Each nucleosynthesis calculation is initiated when the temperature decreases to $10 \mathrm{GK}$, where the initial composition is given by $Y_{\mathrm{e}}$ and $1-Y_{\mathrm{e}}$ for the mass fractions of free protons and neutrons.

The reaction network consists of 6300 species from single neutrons and protons to the $Z=110$ isotopes. Experimental rates, when available, are taken from the latest versions of REACLIB $^{7}$ (Cyburt et al. 2010) and Nuclear Wallet Cards. ${ }^{8}$ Otherwise, the theoretical estimates of fusion rates ${ }^{9}$ (TALYS; Goriely et al. 2008) and $\beta$-decay half-lives (GT2; Tachibana et al. 1990) are adopted, where both are based on the same nuclear masses (HFB-21; Goriely et al. 2010). Theoretical fission properties adopted are those estimated on the basis of the HFB-14 mass model. For fission fragments, a Gaussian-type distribution is assumed with emission of four prompt neutrons per event. Neutrino captures are not included, which make only slight shifts of $Y_{\mathrm{e}}$ (typically an increase of $\sim 0.01$ from $10 \mathrm{GK}$ to 5 GK).

The hydrodynamical trajectories end with temperatures $\sim 5 \mathrm{GK}$. Further temporal evolutions are followed by the density drop such as $t^{-3}$ and with the temperatures computed with the EOS of Timmes \& Swesty (2000) by adding the entropies generated by $\beta$-decay, fission, and $\alpha$-decay. This entropy generation

\footnotetext{
7 https://groups.nscl.msu.edu/jina/reaclib/db/index.php

8 http://www.nndc.bnl.gov/wallet/

9 http://www.astro.ulb.ac.be/pmwiki/Brusslib/Brusslib
} 

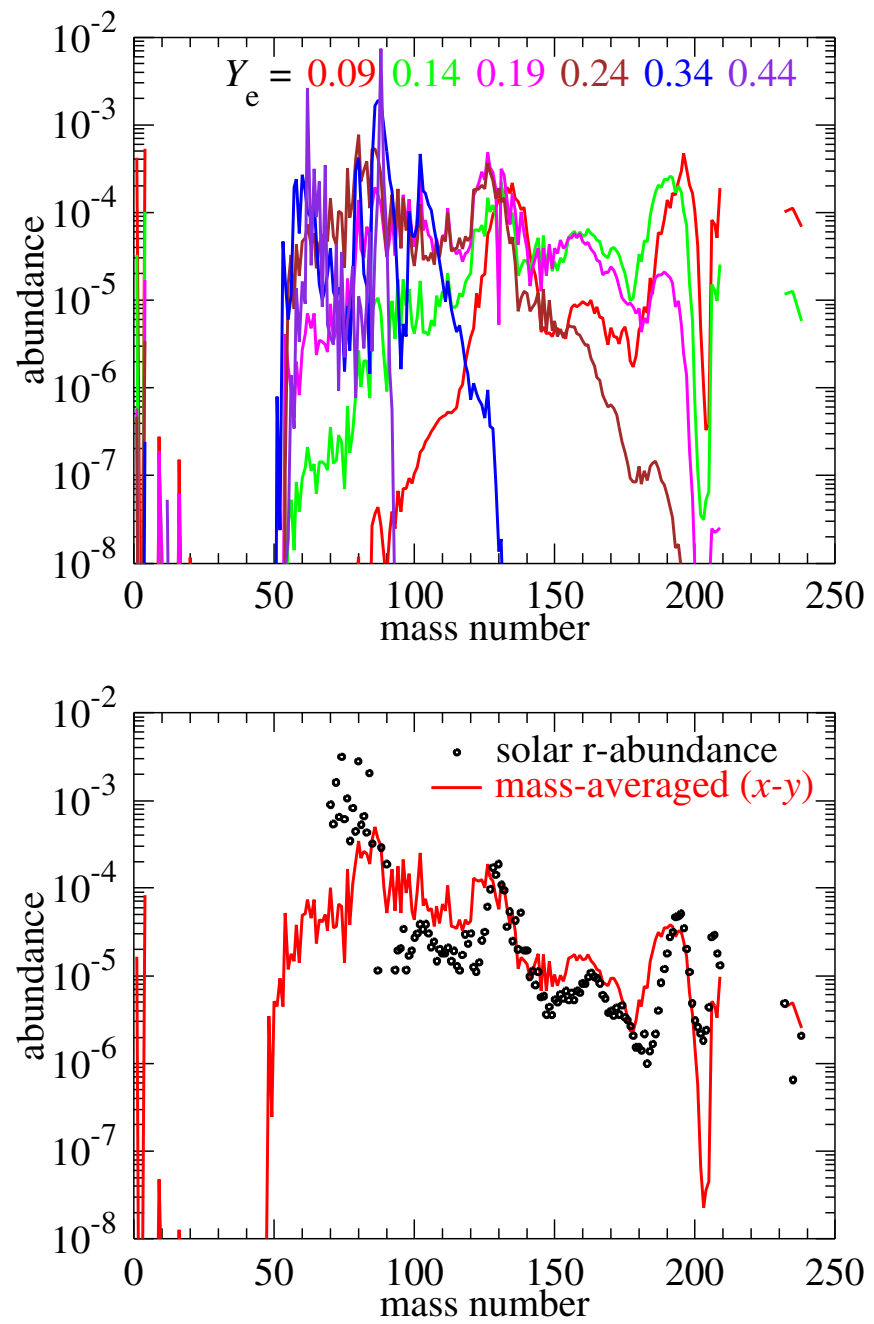

Figure 4. Final nuclear abundances for selected trajectories (top) and that massaveraged (bottom; compared with the solar $r$-process abundances).

(A color version of this figure is available in the online journal.)

slows the temperature drop around $1 \mathrm{GK}$ (e.g., Korobkin et al. 2012). The effect is, however, less dramatic than those found in previous works because of the higher ejecta entropies in our result.

Figure 4 (top) displays the final nuclear abundances for selected trajectories. We find a variety of nucleosynthetic outcomes: iron-peak and $A \sim 90$ abundances made in nuclear quasi-equilibrium for $Y_{\mathrm{e}} \gtrsim 0.4$, light $r$-process abundances for $Y_{\mathrm{e}} \sim 0.2-0.4$, and heavy $r$-process abundances for $Y_{\mathrm{e}} \lesssim 0.2$. In contrast to previous works, we find no fission recycling; the nuclear flow for the lowest $Y_{\mathrm{e}}(=0.09)$ trajectory reaches $A \sim 280$, the fissile point by neutron-induced fission, only at the freezeout of $r$-processing. Spontaneous fission plays a role for forming the $A \sim 130$ abundance peak, but only for $Y_{\mathrm{e}}<0.15$.

Figure 4 (bottom) shows the mass-averaged nuclear abundances by weighting the final yields for the representative trajectories with their $Y_{\mathrm{e}}$ mass fractions on the orbital plane (Figure 3 ). We find a good agreement of our result with the solar $r$-process abundance distribution over the full- $A$ range of 90-240 (although the pattern would be somewhat modified by adding non-orbital components). This result, differing from the previous works exhibiting the production of $A \gtrsim 130 \mathrm{nu}-$ clei only, is a consequence of the wide $Y_{\mathrm{e}}$ distribution predicted from our full GR, neutrino transport simulation. Note also that fission plays a subdominant role for the final nucleosynthetic abundances. The second $(A \sim 130)$ and rare-Earth-element $(A \sim 160)$ peak abundances are dominated by direct production from the trajectories of $Y_{\mathrm{e}} \sim 0.2$. Our result reasonably reproduces the solar-like abundance ratio between the second $(A \sim 130)$ and third $(A \sim 195)$ peaks as well, which is difficult to explain by fission recycling.

Given that the model is representative of NS-NS mergers, our result gives an important implication; the dynamical ejecta of NS-NS mergers can be the dominant origin of all the Galactic $r$-process nuclei. Other contributions from, e.g., the BH-torus wind after collapse of HMNSs, as invoked in the previous studies to account for the (solar-like) $r$-process universality, may not be needed. The amount of entirely $r$-processed ejecta $M_{\mathrm{ej}} \approx 0.01 M_{\odot}$ with present estimates of the Galactic event rate (a few $10^{-5} \mathrm{yr}^{-1}$, e.g., Dominik et al. 2012) is also compatible with the mass of the Galactic r-process abundances as also discussed in previous studies (Korobkin et al. 2012; Bauswein et al. 2013).

\section{RADIOACTIVE HEATING}

The $r$-processing ends a few $100 \mathrm{~ms}$ after the merging. The subsequent abundance changes by $\beta$-decay, fission, and $\alpha$-decay are followed up to $t=100$ days; the resulting radioactive heating is relevant for kilonova emission. Figure 5 displays the temporal evolutions of the heating rates for selected trajectories (top left) and those mass-averaged (top right). For comparison, the heating rate for the nuclear abundances with the solar $r$-process pattern (for $A \geqslant 90, \dot{q}_{\text {solar }-r}$; the same as that used in Hotokezaka et al. 2013b; Tanaka et al. 2014), $\beta$-decaying back from the neutron-rich region, is also shown in each panel. The short-dashed line indicates an analytical approximation defined by $\dot{q}_{\text {analytic }} \equiv 2 \times 10^{10} t^{-1.3}$ (in units of $\mathrm{erg} \mathrm{g}^{-1} \mathrm{~s}^{-1} ; t$ is time in day, e.g., Metzger et al. 2010). The lower panels show the heating rates relative to $\dot{q}_{\text {analytic }}$.

Overall, each curve reasonably follows $\dot{q}_{\text {analytic }}$ by $\sim 1$ day. After this time, the heating is dominated by a few radioactivities and becomes highly dependent on $Y_{\mathrm{e}}$. Contributions from the ejecta of $Y_{\mathrm{e}}>0.3$ are generally unimportant after $\sim 1$ day. We find that the heating for $Y_{\mathrm{e}}=0.34$ turns to be significant after a few tens of days because of the $\beta$-decays from ${ }^{85} \mathrm{Kr}$ (half-life of $T_{1 / 2}=10.8 \mathrm{yr}$; see Figure 4 for its large abundance), ${ }^{89} \mathrm{Sr}$ $\left(T_{1 / 2}=50.5\right.$ days $)$, and ${ }^{103} \mathrm{Ru}\left(T_{1 / 2}=39.2\right.$ days $)$. Heating rates for $Y_{\mathrm{e}}=0.19$ and 0.24 , the abundances of which are dominated by the second peak nuclei, are found to be in good agreement with $\dot{q}_{\text {solar }-r}$. This is due to a predominance of $\beta$-decay heating from the second peak abundances, e.g. ${ }^{123} \operatorname{Sn}\left(T_{1 / 2}=129\right.$ days $)$ and ${ }^{125} \mathrm{Sn}\left(T_{1 / 2}=9.64\right.$ days $)$ around a few tens of days.

Our result shows that the heating rate for the lowest $Y_{\mathrm{e}}$ $(=0.09)$ is the greatest after 1 day with a few times larger values than those in previous works (with $Y_{\mathrm{e}} \sim 0.02-0.04$ in Goriely et al. 2011; Rosswog et al. 2014). In our case, the radioactive heating is dominated by the spontaneous fissions of ${ }^{254} \mathrm{Cf}$ and ${ }^{259,262} \mathrm{Fm}$. It should be noted that the heating from spontaneous fission is highly uncertain because of the many unknown halflives and decay modes of nuclides reaching to this quasi-stable region ( $A \sim 250-260$ with $T_{1 / 2}$ of days to years). In fact, tests with another set of theoretical estimates show a few times smaller rates after $\sim 1$ day (because of diminishing contributions from ${ }^{259,262} \mathrm{Fm}$ ), being similar to the previous works. It appears difficult to obtain reliable heating rates with currently available nuclear data when fission plays a dominant role. 

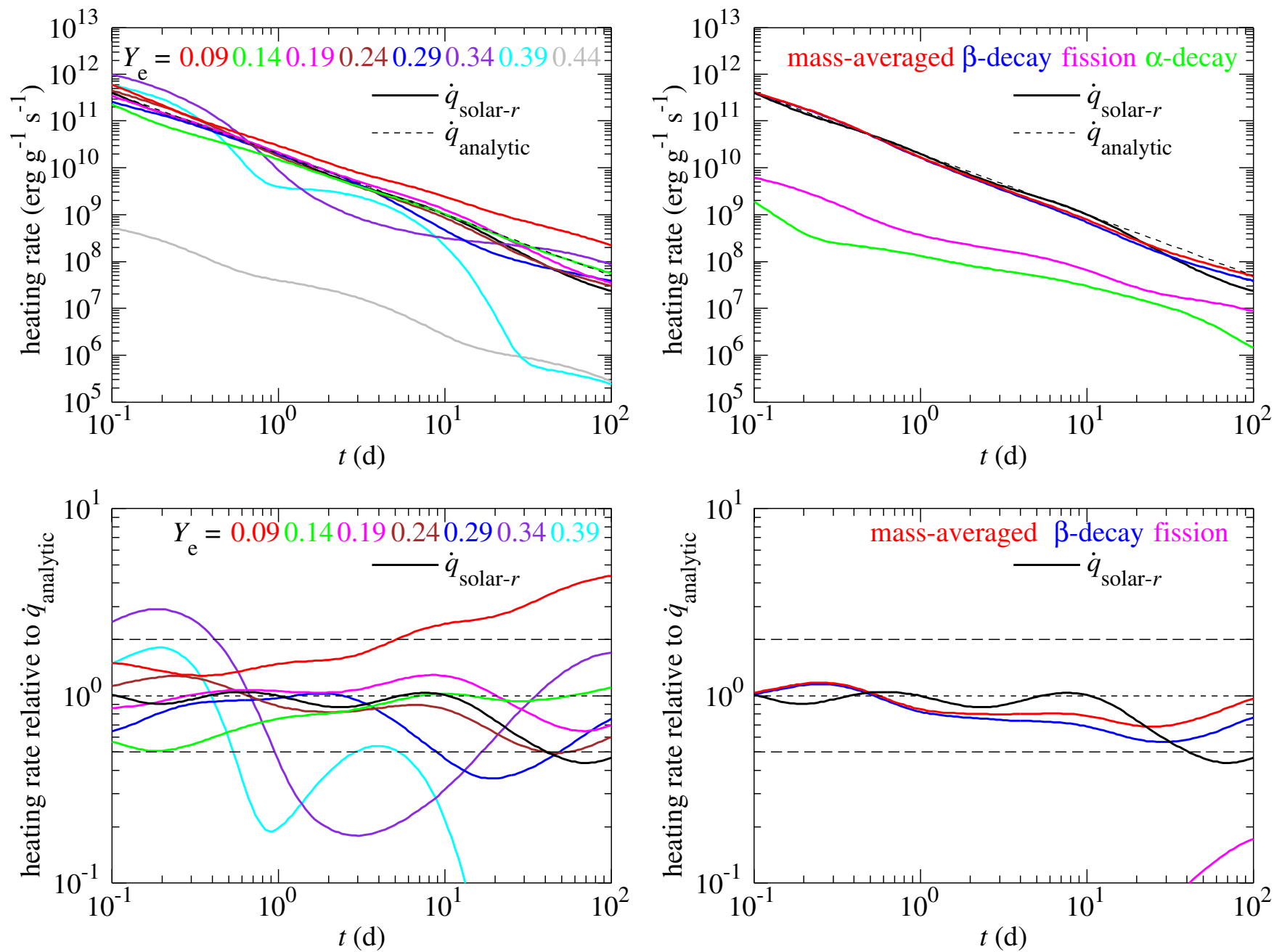

Figure 5. Heating rates as functions of $t$ (days after the merging) for selected trajectories (top left) and those mass-averaged (top right; also shown are those from $\beta$-decay, fission, and $\alpha$-decay). In each panel, the heating rates for the solar $r$-process pattern $\left(\dot{q}_{\text {solar }-r}\right)$ and the analytical approximation $\left(\dot{q}_{\text {analytic }}\right)$ are shown by black-solid and short-dashed lines, respectively. Lower panels are the same as the upper panels but for those relative to $\dot{q}_{\text {analytic }}$. Long-dashed lines indicate the factor of two ranges from unity (short-dashed line).

(A color version of this figure is available in the online journal.)

In our result, the total heating rate is dominated by $\beta$ decays all the time because of the small ejecta amount of $Y_{\mathrm{e}}<0.15$. The radioactive heating after $\sim 1$ day is mostly due to the $\beta$ decays from a small number of species with precisely measured half-lives. Uncertainties in nuclear data are thus irrelevant. The mass-averaged heating rate for $t \sim 1-10$ days is smaller than $\dot{q}_{\text {analytic }}$ and $\dot{q}_{\text {solar }-r}$ because of the overabundances near $A=$ 100 (Figure 4, bottom) that do not significantly contribute to heating. The differences are, however, well within about a factor of two. In conclusion, if merger ejecta have a solar $r$-processlike abundance pattern, $\dot{q}_{\text {solar }-r}$ (and $\dot{q}_{\text {analytic }}$ ) serves as a good approximation for kilonova emission. ${ }^{10}$

It is important to note that our merger simulation exhibits different $Y_{\mathrm{e}}$ distributions between the orbital and polar directions (Figure 3). Multi-dimensional information of nucleosynthetic abundances will be necessary when we discuss the angler

10 These heating rates correspond to the heating efficiency, defined by $f \equiv \dot{Q} t_{\text {peak }} / M_{\text {ej }} c^{2}$ ( $\dot{Q}$, and $t_{\text {peak }}$ are the total heating rate and peak time of a kilonova transient, Li \& Paczyński 1998), of $f / 10^{-6} \approx 1$ and 0.5 for $t_{\text {peak }}=1$ and 10 days, respectively, with the thermalization factor of 0.5 (Metzger et al. 2010). dependences of kilonova emission (Roberts et al. 2011; Grossman et al. 2014).

\section{SUMMARY}

We examined $r$-process calculations based on the full GR, approximate neutrino transport simulation of the NS-NS merger with the equal masses $\left(=1.3 M_{\odot}\right)$ of NSs. In contrast to previous studies, the merger ejecta exhibited a wide range of $Y_{\mathrm{e}} \approx 0.09-0.45$ that led to the nucleosynthetic abundance distribution being in good agreement with the solar $r$-process pattern. Given that the model is representative, our result (with the present estimate of the Galactic event rate) implies that NS-NS mergers can be the major origin of all the $r$-process elements in the Galaxy.

Our result also indicates that the radioactive heating (which powers a kilonova transient) after $\sim 1$ day from the merging is dominated by the $\beta$ decays of a small number of species with measured half-lives. The total heating rates are thus well approximated by the $\beta$ decays of the solar $r$-process-like abundances as well as by the approximation of $\propto t^{-1.3}$. Detailed multi-dimensional information of nucleosynthesis abundances 
should, however, be taken into account when we consider the spatial dependences of kilonova emission.

Our result implies that previous thought on NS-NS merger events, that they dynamically eject almost pure NS matter, should be reconsidered. The shock-heated and neutrinoprocessed ejecta from a HMNS are in fact modestly neutronrich: the phenomenon similar to the early stage of a CCSN (a proto-NS instead of a HMNS). Many more works will be needed to test if similar results are obtained with full 3D nucleosynthetic analyses, with different NS masses and their ratios, with other (reasonable) EOSs, with higher spatial resolution, etc. Nucleosynthetic contributions from BH-NS mergers, as well as from the $\mathrm{BH}$-accretion tori subsequent to NS-NS/BH-NS mergers, should be also explored to draw conclusions on the role of CBMs to the Galactic chemical evolution of the $r$-process nuclei.

We are grateful to S. Goriely and T. Tachibana for providing data of fission properties and $\beta$-decay rates and M. Hempel for the EOS table. The project was supported by the RIKEN iTHES Project, the JSPS Grants-in-Aid for Scientific Research $(23740160,24244028,24740163,25103510,25103512$, 25105508, 26400232, 26400237), Grant-in-Aid for Scientific Research on Innovative Area (20105004), HPCI Strategic Program, and EU-FP7-ERC-2012-St Grant 306901. Koutarou Kyutoku is supported by JSPS Postdoctoral Fellowships for Research Abroad. CBM simulations were in part performed on Cray XC30 at CfCA of NAOJ, Fujitsu FX10 at (Information Technology Center of) the University of Tokyo, and SR16000 at YITP at Kyoto University. This work was in part developed during the long-term workshop on Gravitational Waves and $\mathrm{Nu}$ merical Relativity held at the Yukawa Institute for Theoretical Physics, Kyoto University in 2013 May and June.

\section{REFERENCES}

Antoniadis, J., Freire, P. C. C., Wex, N., et al. 2013, Sci, 340, 6131

Barnes, J., \& Kasen, D. 2013, ApJ, 775, 18

Bauswein, A., Goriely, S., \& Janka, H.-T. 2013, ApJ, 773, 78

Berger, E., Fong, W., \& Chornock, R. 2013, ApJL, 774, L23

Cyburt, R. H., Amthor, A. M., Ferguson, R., et al. 2010, ApJS, 189, 240

Demorest, P. B., Pennucci, R., Ransom, S. M., Roberts, M. S. E., \& Hessels, J. W. T. 2010, Natur, 467, 1081

Dessart, L., Ott, C. D., Burrows, A., Rosswog, S., \& Livne, E. 2009, ApJ, 690,1681
Dominik, M., Belczynski, K., Fryer, C., et al. 2012, ApJ, 759, 52

Eichler, D., Livio, M., Piran, T., \& Schramm, D. N. 1989, Natur, 340, 126

Fernández, R., \& Metzger, B. D. 2013, MNRAS, 435, 502

Fischer, T., Martínez-Pinedo, G., Hempel, M., \& Liebendörfer, M. 2012, PhRvD, 85,083003

Freiburghaus, C., Rosswog, S., \& Thielemann, F.-K. 1999, ApJL, 525, L121

Goriely, S., Chamel, N., Janka, H.-T., \& Pearson, J. M. 2011, A\&A, 531, A78

Goriely, S., Chamel, N., \& Pearson, J. M. 2010, PhRvC, 82, 035804

Goriely, S., Hilaire, S., \& Koning, A. J. 2008, A\&A, 487, 767

Grossman, D., Korobkin, O., Rosswog, S., \& Piran, T. 2014, MNRAS, 439, 757

Hotokezaka, K., Kiuchi, K., Kyutoku, K., et al. 2013a, PhRvD, 87, 024001

Hotokezaka, K., Kyutoku, K., Tanaka, M., et al. 2013b, ApJL, 778, L16

Kasen, D., Badnell, N. R., \& Barnes, J. 2013, ApJ, 774, 25

Korobkin, O., Rosswog, S., Arcones, A., \& Winteler, C. 2012, MNRAS, 426, 1940

Lattimer, J. M., \& Lim, Y. 2013, ApJ, 771, 51

Lattimer, J. M., \& Schramm, D. N. 1974, ApJL, 192, L145

Li, L.-X., \& Paczyński, B. 1998, ApJL, 507, L59

Martínez-Pinedo, G., Fischer, T., Lohs, A., \& Huther, L. 2012, PhRvL, 109, 251104

Metzger, B. D., Martínez-Pinedo, G., Darbha, S., et al. 2010, MNRAS, 406, 2650

Meyer, B. S. 1989, ApJ, 343, 254

Qian, Y.-Z., \& Woosley, S. E. 1996, ApJ, 471, 331

Roberts, L. F., Kasen, D., Lee, W. H., \& Ramirez-Ruiz, E. 2011, ApJL, 736, L21

Roberts, L. F., Reddy, S., \& Shen, G. 2012, PhRvC, 86, 065803

Rosswog, S., Korobkin, O., Arcones, A., \& Thielemann, F.-K. 2014, MNRAS, 439, 744

Sekiguchi, Y. 2010, PThPh, 124, 331

Sekiguchi, Y., Kiuchi, K., Kyutoku, K., \& Shibata, M. 2011a, PhRvL, 107,051102

Sekiguchi, Y., Kiuchi, K., Kyutoku, K., \& Shibata, M. 2011b, PhRvL, 107,211101

Shen, H., Toki, H., Oyamatsu, K., \& Sumiyoshi, K. 1998, NuPhA, 637, 435

Shibata, M., Kiuchi, K., Sekiguchi, Y., \& Suwa, Y. 2011, PThPh, 125, 1255

Siqueira Mello, C., Hill, V., Barbuy, B., et al. 2014, A\&A, 565, A93

Sneden, C., Cowan, J. J., \& Gallino, R. 2008, ARA\&A, 46, 241

Steiner, A. W., Hempel, M., \& Fischer, T. 2013, ApJ, 774, 17

Surman, R., McLaughlin, G. C., Ruffert, M., Janka, H.-Th., \& Hix, W. R. 2008, ApJL, 679, L117

Symbalisty, E., \& Schramm, D. N. 1982, ApL, 22, 143

Tachibana, T., Yamada, M., \& Yoshida, Y. 1990, PThPh, 84, 641

Tanaka, M., \& Hotokezaka, K. 2013, ApJ, 775, 113

Tanaka, M., Hotokezaka, K., Kyutoku, K., et al. 2014, ApJ, 780, 31

Tanvir, N. R., Levan, A. J., Fruchter, A. S., et al. 2013, Natur, 500, 547

Thorne, K. S. 1981, MNRAS, 194, 439

Timmes, F. X., \& Swesty, F. D. 2000, ApJS, 126, 501

Wanajo, S. 2013, ApJL, 770, L22

Wanajo, S., \& Janka, H.-T. 2012, ApJ, 746, 180

Wanajo, S., Janka, H.-T., \& Müller, B. 2011, ApJL, 726, L15

Winteler, C., Käppeli, R., Perego, A., et al. 2012, ApJL, 750, L22 